\title{
Functional MRI Motor Imagery Tasks to Detect Command Following in Traumatic Disorders of Consciousness
}

\begin{abstract}
Yelena G. Bodien ${ }^{1,2 *}$, Joseph T. Giacino ${ }^{2,3}$ and Brian L. Edlow ${ }^{1,4}$
${ }^{1}$ Center for Neurotechnology and Neurorecovery, and Laboratory for Neurolmaging of Coma and Consciousness, Department of Neurology, Massachusetts General Hospital, Harvard Medical School, Boston, MA, United States,

${ }^{2}$ Department of Physical Medicine and Rehabilitation, Spaulding Rehabilitation Hospital, Harvard Medical School, Boston, MA, United States, ${ }^{3}$ Department of Psychiatry, Massachusetts General Hospital and Harvard Medical School, Boston, MA, United States, ${ }^{4}$ Athinoula A. Martinos Center for Biomedical Imaging, Massachusetts General Hospital and Harvard Medical School, Charlestown, MA, United States
\end{abstract}

Severe traumatic brain injury impairs arousal and awareness, the two components of consciousness. Accurate diagnosis of a patient's level of consciousness is critical for determining treatment goals, access to rehabilitative services, and prognosis. The bedside behavioral examination, the current clinical standard for diagnosis of disorders of consciousness, is prone to misdiagnosis, a finding that has led to the development of advanced neuroimaging techniques aimed at detection of conscious awareness. Although a variety of paradigms have been used in functional magnetic resonance imaging (fMRl) to reveal covert consciousness, the relative accuracy of these paradigms in the patient population is unknown. Here, we compare the rate of covert consciousness detection by hand squeezing and tennis playing motor imagery paradigms in 10 patients with traumatic disorders of consciousness [six male, six acute, mean \pm SD age $=27.9 \pm 9.1$ years, one coma, four unresponsive wakefulness syndrome, two minimally conscious without language function, and three minimally conscious with language function, per bedside examination with the Coma Recovery Scale-Revised (CRS-R)]. We also tested the same paradigms in 10 healthy subjects (nine male, mean $\pm \mathrm{SD}$ age $=28.5 \pm 9.4$ years). In healthy subjects, the hand squeezing paradigm detected covert command following in $7 / 10$ and the tennis playing paradigm in $9 / 10$ subjects. In patients who followed commands on the CRS-R, the hand squeezing paradigm detected covert command following in 2/3 and the tennis playing paradigm in $0 / 3$ subjects. In patients who did not follow commands on the CRS-R, the hand squeezing paradigm detected command following in 1/7 and the tennis playing paradigm in 2/7 subjects. The sensitivity, specificity, and accuracy (ACC) of detecting covert command following in patients who demonstrated this behavior on the CRS-R was $66.7,85.7$, and $80 \%$ for the hand squeezing paradigm and $0,71.4$, and $50 \%$ for the tennis playing paradigm, respectively. Overall, the tennis paradigm performed better than the hand squeezing paradigm in healthy subjects, but in patients, the hand squeezing paradigm detected command following with greater ACC. These findings indicate that current fMRI motor imagery paradigms frequently 
fail to detect command following and highlight the need for paradigm optimization to improve the accuracy of covert consciousness detection.

Keywords: traumatic brain injury, consciousness, awareness, functional magnetic resonance imaging, motor imagery

\section{INTRODUCTION}

Patients with severe traumatic brain injury (TBI) experience a period of impaired consciousness characterized by disturbances in arousal and awareness. This disorder of consciousness (DoC) may resolve acutely [i.e., in the intensive care unit (ICU)] or may be prolonged, extending weeks, months, or even years postinjury (1). The spectrum of behavioral states that comprise DoC includes coma, unresponsive wakefulness syndrome (UWS, also known as vegetative state) (2,3), minimally conscious state (MCS) (4), and post-traumatic confusional state $(5,6)$. Accurate assessment of a patient's level of consciousness (LoC) is critically important to prognosis, as patients who have recovered consciousness (i.e., MCS) and especially language function have a higher likelihood of regaining cognitive function than those who have not (i.e., coma and UWS) (7-10). Thus, assessment of LoC drives early decisions about aggressive treatment and access to rehabilitative care. An inaccurate diagnosis may also prevent autonomous decision-making in patients who retain the capacity to do so.

Despite the critical importance of accurately defining a patient's LoC, the current standard for assessment in this patient population is bedside clinical examination, a method that is prone to inaccuracies due to patient impairments (e.g., speech and motor deficits that prevent verbalization or movement to command) and examiner bias. The approximate rate of misdiagnosing a conscious patient as unconscious is $40 \%$ (11-14). Standardized behavioral tools, such as the Coma Recovery Scale-Revised (CRS-R) (15), have helped improve the accuracy and precision of the bedside assessment, but the behavioral diagnosis is potentially susceptible to misinterpretation of subtle and inconsistent behaviors. Recently published guidance on the optimal frequency of CRS-R assessment may further improve the accuracy of behavioral assessment (16), but even frequent assessments with the CRS-R may fail to detect consciousness in persons whose capacity for volitional brain function is masked by limitations in self-expression. Objective markers of consciousness are therefore needed to ensure accurate diagnosis and to guide care management.

To circumvent some of the limitations of the bedside behavioral examination, recent studies have attempted to elicit evidence of consciousness by asking a patient to perform a mental imagery task in a magnetic resonance imaging (MRI) scanner

Abbreviations: DoC, disorders of consciousness; fMRI, functional magnetic resonance imaging; ICU, intensive care unit; LoC, level of consciousness; MCS \pm , minimally conscious state plus/minus; PTCS, post-traumatic confusional state; TBI, traumatic brain injury; UWS, unresponsive wakefulness syndrome.
(17-24). These functional MRI (fMRI) motor imagery tasks are not confounded by speech or motor impairment and therefore may provide additional information about a patient's LoC that cannot be obtained by a behavioral assessment. Moreover, the magnitude, signal characteristics, and neuroanatomic location of brain activations detected by fMRI can be analyzed using predetermined objective algorithms that are independent of observer bias or variations in the administration and scoring of standardized behavioral measures. Several fMRI studies have identified persons with acute $(24)$ and chronic $(21,25)$ DoC who demonstrate cognitive-motor dissociation (CMD) (26), which is defined by fMRI evidence of command following in the absence of behavioral evidence of command following.

Currently, there is a lack of consensus regarding which fMRI paradigms are best suited to elicit covert command following and hence a diagnosis of CMD. Although early studies used covert object naming (21), and some have employed covert counting of target words (27), most recent fMRI investigations have focused on spatial navigation and motor imagery tasks (e.g., imagine playing tennis, swimming, or squeezing the right or left hand) (17). For a review of tasks used to elicit command following in patients with DoC see Rossetti and Laureys (28) and Laureys and Schiff (29). In 2007, Boly and colleagues (30) compared the robustness of brain activation to four task-based fMRI imagery paradigms in healthy subjects: spatial navigation (imagine walking around the rooms of a house), auditory imagery (imagine a familiar song), motor imagery (imagine hitting a tennis ball), and visual imagery (imagine familiar faces). They found that the spatial navigation and tennis imagery tasks provided the most robust results in healthy subjects. Consequently, tennis motor imagery has been utilized frequently over the past decade to identify CMD in patients with DoC.

Although the fMRI tennis imagery task seems to be a viable complement to the bedside examination of patients with DoC, several studies have found high false-negative rates (FNRs) using this task (i.e., patient and healthy subjects who have behavioral evidence of command following do not demonstrate the expected activations on tennis imagery fMRI tasks) $(17,31)$. A hand squeezing motor imagery task has been used successfully in EEG studies $(32,33)$ and may be a more robust paradigm for use in the ICU, as it parallels the clinical bedside examination and may be less cognitively burdensome than imagining playing tennis. It remains unknown whether tennis playing imagery or hand squeezing imagery is a more effective paradigm for detecting conscious awareness.

Our aim in the present study was to compare fMRI activation in response to a tennis playing and hand squeezing paradigm in patients with traumatic DoC. The hand squeezing paradigm was 
chosen because it is a simple motor response that closely resembles the bedside behavioral examination, which often includes a "squeeze my hand" instruction to elicit command following. In addition, hand squeezing is more universal than playing tennis, which may be imagined differently and with varying intensity depending on an individual's exposure to the sport. The hand squeezing task has been used successfully in other studies in acute and chronic DoC $(24,32,33)$. We hypothesized that hand squeezing motor imagery will be detected with greater frequency than tennis playing motor imagery in patients diagnosed with acute and chronic traumatic DoC and in healthy controls.

\section{MATERIALS AND METHODS}

\section{Experimental Design}

This study was carried out in accordance with a protocol approved by the Partners Institutional Review Board. Patient surrogate decision-makers gave written informed consent in accordance with the Declaration of Helsinki. The patient cohort was prospectively recruited from an ICU, an outpatient follow-up neurology clinic, and an affiliated long-term acute hospital. Inclusion criteria were as follows: (1) age 18-65 years; and (2) head trauma with Glasgow Coma Scale score of 3-8 with no eye opening for at least $24 \mathrm{~h}$ after injury. Exclusion criteria were as follows: (1) life expectancy less than 6 months, as determined by a treating clinician; (2) prior severe brain injury or neurodegenerative disease; (3) penetrating TBI with intracranial metal or other body metal precluding MRI; and (4) no fluency in English prior to the injury (because the paradigms were administered in English).

Surrogate decision-makers were approached for consent $\geq 24 \mathrm{~h}$ after injury. For the ICU cohort, fMRI was performed as soon as the patient was clinically stable for transport to the MRI scanner, as determined by the treating ICU physicians and nurses. Patients with chronic DoC were scanned when they returned to the hospital for an outpatient clinic appointment or an inpatient hospitalization (e.g., for cranioplasty). Administration of sedative, anxiolytic, and/or analgesic medications was permitted for patient safety or comfort, at the discretion of the treating clinicians.

Age-matched healthy subjects were enrolled as a comparison group. Healthy subjects had no history of neurological, psychiatric, cardiovascular, pulmonary, renal, or endocrinological disease. They provided written informed consent and underwent the same fMRI protocols as the patients. All patient and healthy subject MRI scans were performed on the same scanner.

\section{Neurobehavioral Assessment}

Demographic and clinical data were collected at the time of enrollment in accordance with the National Institutes of Health Common Data Element Guidelines for TBI. ${ }^{1}$ LoC was characterized via behavioral evaluation with the CRS-R or based on criteria derived from the Confusion Assessment Protocol immediately prior to the fMRI $(6,34)$. Based on the CRS-R, each patient's LoC was defined as coma (no arousal or

${ }^{1}$ https://www.commondataelements.ninds.nih.gov. awareness), UWS (return of arousal but no awareness of self or the environment) $(2,3)$, or MCS (return of arousal and reliable but inconsistent evidence of awareness) (4). MCS was further subdivided into MCS- and MCS+ with the distinguishing feature being the presence of language function (i.e., at least one of the following: command following, object-recognition, or intelligible verbalization) in patients diagnosed as MCS + (35, 36). Emergence from MCS was marked by recovery of either functional use of two common objects or basic accurate communication. The neurobehavioral assessment was conducted immediately prior to the fMRI scan. All behavioral evaluations were conducted by Brian L. Edlow.

\section{MRI Data Acquisition}

Magnetic resonance imaging data were acquired with a 32-channel head coil on a $3 \mathrm{~T}$ Siemens Skyra MRI scanner (Siemens Medical Solutions, Erlangen, Germany) located in the Neurosciences ICU. Auditory stimuli were presented via MRIcompatible earphones (Newmatic Medical, Caledonia, MI, USA) connected to the scanner's sound system. The blood-oxygen level dependent (BOLD) fMRI sequence utilized the following parameters: echo time $(\mathrm{TE})=30 \mathrm{~ms}$, repetition time $(\mathrm{TR})=4,000 \mathrm{~ms}$, in-plane resolution $=2.0 \mathrm{~mm} \times 2.0 \mathrm{~mm}$, slice thickness $=2 \mathrm{~mm}$, interslice gap $=2.5 \mathrm{~mm}$, matrix $=94 \times 94$, field of view $($ FOV $)=192 \mathrm{~mm} \times 192 \mathrm{~mm}, 49$ slices, $2 \times$ GRAPPA acceleration. Image acquisition parameters differed for one subject (P10) due to a change in the fMRI protocol motivated by decreasing scan time. For this subject, the fMRI sequence TE was reduced to $25 \mathrm{~ms}$ and TR to 3,000 ms. High-spatial resolution 3D T1-weighted multiecho magnetization prepared gradient echo (MEMPRAGE) anatomical images were acquired for registration purposes (37): FOV $=256 \mathrm{~mm} \times 256 \mathrm{~mm}$, acquisition matrix $=256 \times 256$, 176 sagittal slices (thickness $1 \mathrm{~mm}$ ), $3 \times$ GRAPPA acceleration, $\mathrm{TE}=1.69,3.55,5.41$, and $7.27 \mathrm{~ms}$, $\mathrm{TR}=2,530 \mathrm{~ms}$, inversion time $=1,200-1,300 \mathrm{~ms}, 1.0 \mathrm{~mm}^{3}$ isotropic resolution, and flip angle $=7^{\circ}$.

\section{fMRI Paradigms}

Two fMRI motor imagery paradigms - hand squeezing and tennis playing - were performed as part of a larger fMRI and EEG study. fMRI data from the hand squeeze task have been previously reported for P1-5 and C1-10 (24). Each motor imagery fMRI paradigm utilized a block design and was comprised of two runs, with each run containing three 24-s rest blocks and two 24-s stimulation blocks. In total, $144 \mathrm{~s}$ of rest data and $96 \mathrm{~s}$ of stimulation data were analyzed for each paradigm. Prior to the first rest block, $36 \mathrm{~s}$ of data ( $9 \mathrm{~s}$ for P10) were acquired to obtain a stable baseline BOLD signal. These data were excluded from analysis.

The hand squeeze motor imagery task always preceded the tennis motor imagery task because the former paradigm was part of the primary aim of a larger study (24) and the latter paradigm was added as part of a secondary study aim after initiation of data collection. Similar to other studies in $\operatorname{DoC}(32,33)$, subjects were instructed to imagine squeezing their right hand or to rest. During the task, instructions to "keep squeezing" or to "keep resting" were repeated at 6-s intervals. The tennis playing task 
was identical to the hand squeezing task except that subjects were instructed to imagine playing tennis or to rest. During the task, instructions of "keep playing" or "keep resting" were repeated at 6-s intervals. Instructions administered before and during the fMRI are detailed in Table S1 in Supplementary Material.

\section{fMRI Data Analysis}

In a first-level analysis of the individual runs, fMRI data processing was performed using the FMRI Expert Analysis Tool (FEAT) version 6.00 in FSL 5.0.7 (FMRIB's Software Library ${ }^{2}$ ). Motor imagery stimuli were contrasted against rest. $Z$ statistic images

$\overline{{ }^{2} \text { www.fmrib.ox.ac.uk/fsl. }}$.

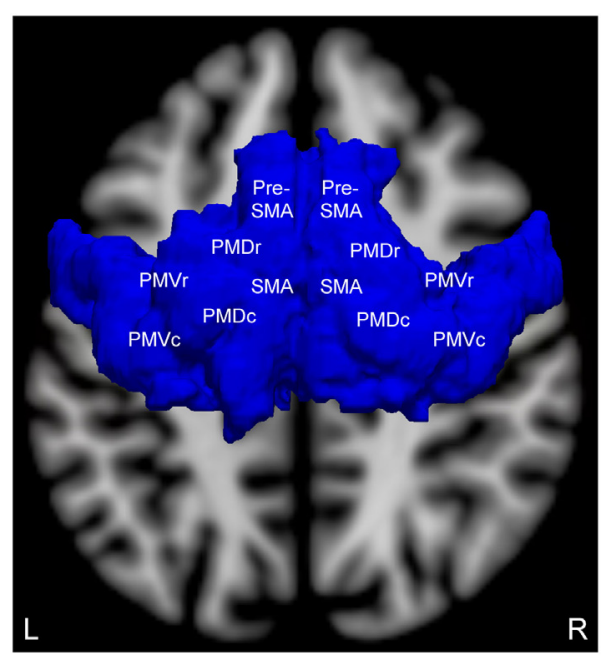

FIGURE 1 | Brodmann area 6 region of interest (superior view) for measuring motor imagery functional magnetic resonance imaging (fMRI) activation. This region of interest contains the supplementary motor area (SMA), pre-SMA, and the four components of the bilateral premotor cortices: premotor dorsal rostral (PMDr), premotor dorsal caudal (PMDc), premotor ventral rostral (PMVr), and premotor ventral caudal (PMVc). Adapted and reproduced with permission from Edlow et al. (24). were thresholded $(Z>3.1)$ and a corrected cluster significance threshold of $P=0.05$ was used. Higher-level analysis was carried out using a fixed effects model (FLAME in FSL) $(38,39)$. The statistical threshold for cluster significance $(Z>3.1)$ and the size of the Gaussian kernel (FWHM $10 \mathrm{~mm}$ ) were both selected to decrease false positive cluster activations (40). Additional details on analysis have been published elsewhere (24).

We used FEATQuery in FSL to quantify the percentage of voxels activated within a prespecified region of interest (ROI). For healthy subjects, we defined a positive response by the criterion that $>0 \%$ of ROI voxels met the aforementioned statistical threshold. For patients, we defined a positive response by two criteria, consistent with a recently proposed definition (24): (1) $>0 \%$ of ROI voxels met the statistical threshold; and (2) the percentage of activated ROI voxels was above the 2.5 th percentile of a normal range (2.5th-97.5th percentile) derived from the healthy subjects' data for each paradigm. This quantitative approach was completely automated and did not require subjective interpretation by clinical or research staff, thereby reducing potential bias that may have been introduced by knowledge of the patient's behavioral diagnosis.

\section{fMRI Regions of Interest}

We selected an a priori ROI based upon fMRI studies of motor imagery in patients with chronic traumatic DoC and healthy subjects, as well as a recently published study of patients with acute traumatic DoC that used this same ROI (24). The bilateral supplementary motor areas (SMA) from the Harvard-Oxford Cortical Structural Atlas and premotor cortices (PMC) from the Juelich Histological Atlas (41) were combined as a single ROI (Figure 1). This ROI was transformed from standard atlas space into patient native fMRI space for analysis, consistent with prior fMRI studies of patients with $\operatorname{DoC}(17,20,24,42,43)$.

\section{Statistical Analysis}

Using the CRS-R as the reference standard and the motor imagery fMRI responses as the test criteria, we assessed the reliability of each paradigm in detecting behavioral evidence of command following by calculating the true-positive rate (TPR;

\begin{tabular}{|c|c|c|c|c|c|c|c|c|c|c|}
\hline ID & $\mathrm{C}{ }^{++}$ & $\mathrm{C}^{++}$ & $\mathrm{C}^{++}$ & $\mathrm{C} 4^{+-}$ & $\mathrm{C} 5^{++}$ & $\mathrm{C}^{-+}$ & $\mathrm{C} 7^{+}$ & $\mathrm{C}^{++}$ & $\mathrm{C} 9^{-}$ & $\mathrm{C} 10^{++}$ \\
\hline Age \& Sex & $20 \mathrm{M}$ & $50 \mathrm{M}$ & $23 \mathrm{~F}$ & $21 \mathrm{M}$ & $27 \mathrm{M}$ & $29 M$ & $21 \mathrm{M}$ & $24 \mathrm{M}$ & $38 \mathrm{M}$ & $32 \mathrm{M}$ \\
\hline $\begin{array}{c}\text { Hand } \\
\text { Squeezing } \\
3.1 \square 6\end{array}$ & & & & & & & & & & \\
\hline $\begin{array}{l}\text { Tennis } \\
\text { Playing }\end{array}$ & & & & & & & & & & \\
\hline
\end{tabular}

FIGURE 2 | Stimulus-based functional magnetic resonance imaging (fMRI) responses to hand squeezing and tennis playing motor imagery paradigms in healthy subjects. fMRI data are shown as Z-statistic images to demonstrate stimulus-specific responses. Z-Statistic images are thresholded at cluster-corrected $Z$ scores of 3.1 (inset color bars) and superimposed upon T1-weighted axial images. In the row-labeled "ID," a "+" indicates that an fMRI response was detected and a "-" indicates that an fMRI response was not detected during the hand squeezing and tennis playing motor imagery paradigms, respectively. Abbreviations: $\mathrm{F}$, female; M, male. All images are in radiologic convention. 


\begin{tabular}{|c|c|c|c|c|c|c|c|c|c|c|}
\hline ID & P1" & $\mathrm{P}^{+*}$ & $\mathrm{P}^{+*}$ & $\mathrm{P}^{-{ }^{+}}$ & $\mathrm{P}^{+}$ & P6" & $\mathrm{P}^{+{ }^{+}}$ & P8" & $\mathrm{P9}^{-}$ & P10"- \\
\hline Age \& Sex & $27 \mathrm{~F}$ & $18 \mathrm{M}$ & $51 \mathrm{M}$ & $29 \mathrm{M}$ & $33 \mathrm{M}$ & $25 \mathrm{M}$ & $22 \mathrm{~F}$ & $26 \mathrm{~F}$ & $26 \mathrm{M}$ & $22 \mathrm{~F}$ \\
\hline Day of MRI & 8 & 4 & 8 & 7 & 3 & 186 & 162 & 12 & 142 & 1900 \\
\hline LoC at MRI & Coma & MCS+ & UWS & MCS- & MCS+ & MCS+ & UWS & UWS & MCS- & UWS \\
\hline $\begin{array}{c}\text { Hand } \\
\text { Squeezing } \\
3.1\end{array}$ & & & & & & & & & & \\
\hline $\begin{array}{l}\text { Tennis } \\
\text { Playing }\end{array}$ & & & & & & & & & & \\
\hline
\end{tabular}

FIGURE 3 | Stimulus-based functional magnetic resonance imaging (fMRI) responses to hand squeezing and tennis playing motor imagery paradigms in patients. fMRI data are shown as Z-statistic images to demonstrate stimulus-specific responses. Z-Statistic images are thresholded at cluster-corrected Z scores of 3.1 (inset color bars) and superimposed upon T1-weighted axial images. Level of consciousness (LoC) is assessed via behavioral evaluation with the Coma Recovery Scale-Revised as coma, unresponsive wakefulness syndrome (UWS), minimally conscious state without language (MCS-), or minimally conscious state with language (MCS+). In the row-labeled "ID," a "+" indicates that an fMRI response was detected and a "-" indicates that an fMRI response was not detected during the hand squeezing and tennis playing motor imagery paradigms, respectively. Abbreviations: F, female; M, male. All images are in radiologic convention.

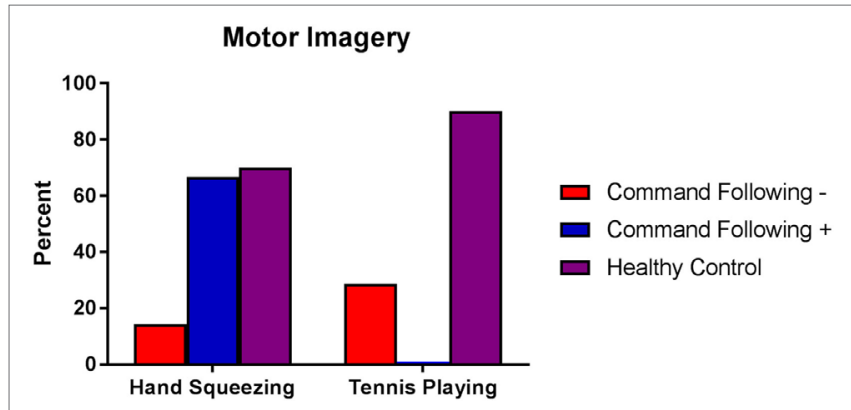

FIGURE 4 | Percentage of healthy subjects, patients without behavioral evidence of command following [command following-(coma, UWS, MCS-)] and patients with behavioral evidence of command following [command following + (MCS+)] who showed functional MRI responses to hand squeezing and tennis playing motor imagery tasks. Behavioral evaluation was performed using the Coma Recovery Scale-Revised. Healthy subjects are represented by purple bars, patients without behavioral evidence of command following by red bars, and patients with behavioral evidence of command following by blue bars. Abbreviations: UWS, unresponsive wakefulness syndrome; MCS-, minimally conscious state without language; MCS+, minimally conscious state with language; MRI, magnetic resonance imaging.

i.e. sensitivity), true-negative rate (TNR; i.e. specificity), FNR and the false-positive rate (FPR) with 95\% exact confidence interval (CI) in the patient cohort. Notably, the FPR may include both false positives (i.e., patients wrongly identified on $\mathrm{fMRI}$ as being able to follow commands) and cases of dissociation between behavioral and fMRI responses (i.e., CMD caused by speech or motor impairments or other confounding factors). The accuracy (ACC) of each fMRI paradigm for detecting command following was calculated as $(\mathrm{TP}+\mathrm{TN}) /(\mathrm{TP}+\mathrm{FP}+\mathrm{TN}+\mathrm{FN})$. We also calculated the TP proportions in the healthy subject cohort but not the other metrics because all healthy subjects exhibited behavioral evidence of command following.

We tested for an association between sedation (dichotomized as presence or absence) and fMRI responses, as well as between sedation and LoC at the time of fMRI (dichotomized as presence or absence of command following), using a $2 \times 2$ Fisher's Exact Test. Statistical analyses were performed in STATA v14s (44).

\section{RESULTS}

\section{Demographics and Clinical Characteristics}

Hand squeeze and tennis playing fMRI data were acquired in a convenience sample of 12 patients. One of these subjects was excluded due to severe artifact resulting from a ventriculoperitoneal shunt that prevented spatial normalization of the data. A second subject was excluded due to errors in the data acquisition of DICOM images. The final patient cohort included 10 subjects ( six male, mean \pm age $=27.9 \pm 9.1$, six acute, one coma, four UWS, two MCS-, and three MCS+). Demographic and clinical data are presented in Table 1. Ten age-matched healthy subjects were recruited (nine male, mean \pm SD age $=28.5 \pm 9.4$, see Table S2 in Supplementary Material). There was no statistical difference in the proportion of males to females (chi-squared, $P>0.10$ ) or in the age of the subjects ( $t$-test, $P>0.88$ ) between the patient and healthy subject groups.

\section{Hand Squeezing}

Seven of the 10 healthy subjects demonstrated covert command following via the hand squeeze paradigm [70\%, 95\% exact CI: (34.8-93.3\%), see Table 2, Figures 2 and 4. Three of the 10 patients demonstrated command following on the hand squeeze paradigm [30\% (6.7-65.3), see Table 3, Figures 3 and 4. Of the three patients with behavioral evidence of command following on bedside examination, two demonstrated command following on the hand squeezing fMRI task $(\mathrm{TP}=2 / 3, \mathrm{FN}=1 / 3)$. Of the seven patients without behavioral evidence of command following, one demonstrated command following on the hand squeeze paradigm $(\mathrm{TN}=6 / 7, \mathrm{FP}=1 / 7)$. Consequently, the sensitivity and specificity of the hand squeeze paradigm for detecting behavioral evidence of command following were $66.7 \%$ (12.5-98.2) and $85.7 \%$ (42.1-99.6), respectively, and ACC $=80 \%$. 
TABLE 1 | Patient demographics and clinical characteristics.

\begin{tabular}{|c|c|c|c|c|c|c|c|c|}
\hline ID & Age (years) & Sex & TBI mechanism & iGCS & Day of fMRI & CRS-R at fMRI & CRS-R subscale scores at fMRI & LoC at fMRI \\
\hline P1 & 27 & $\mathrm{~F}$ & Fall & 3 & 8 & 1 & AOVOM1O0C0ArO & Coma \\
\hline $\mathrm{P} 2$ & 18 & $M$ & Fall & $3-7$ & 4 & 12 & A3V2M501C0Ar1 & MCS+ \\
\hline P3 & 51 & $\mathrm{M}$ & Ped vs. car & 3 & 8 & 3 & AoV0M1O1C0Ar1 & UWS \\
\hline P4 & 29 & $M$ & Ped vs. car & $4-7$ & 7 & 3 & AOV0M300C0ArO & MCS- \\
\hline P5 & 33 & $\mathrm{M}$ & Fall & $3-4$ & 3 & 12 & A4V2M500C0Ar1 & MCS+ \\
\hline P6 & 25 & $M$ & MVA & $3-6 \mathrm{~T}$ & 183 & 15 & A4V3M3O2C1Ar2 & $\mathrm{MCS}+$ \\
\hline P7 & 22 & $\mathrm{~F}$ & Ped vs. car & $3-3 T$ & 162 & 5 & A1V1M1O1C0Ar1 & UWS \\
\hline P8 & 26 & $\mathrm{~F}$ & Ped vs. truck & $3-3 T$ & 12 & 2 & AoV0M100C0Ar1 & UWS \\
\hline P9 & 26 & M & MVA & 3 & 142 & 8 & A1V1M3O2COAr1 & MCS- \\
\hline P10 & 22 & $\mathrm{~F}$ & MVA & 3 & 1,900 & 5 & A1V0M2O1C0Ar1 & UWS \\
\hline
\end{tabular}

The initial GCS (iGCS) is a range defined by the best (i.e., highest) and worst (i.e., lowest) post-resuscitation GCS scores assessed by a qualified clinician who performed a reliable examination (not confounded by sedation and/or paralytics) prior to ICU admission. LoC is assessed immediately prior to fMRI via behavioral evaluation with the CRS-R as coma, UWS, MCS-, or MCS+. The subscales for the CRS-R are auditory function (A), visual function (N), motor function (M), oromotor function (O), communication (C), and arousal (Ar). CRS-R, Coma Recovery Scale-Revised; F, female; fMRI, functional MRI; GCS, Glasgow Coma Scale; LoC, level of consciousness; M, male; MCS-, minimally conscious state without language; MCS+, minimally conscious state with language; MVA, motor vehicle accident; Ped, pedestrian; T, intubated, TBI, traumatic brain injury; UWS, unresponsive wakefulness syndrome.

TABLE 2 | Healthy control subject fMRI responses to hand squeeze and tennis motor imagery.

\begin{tabular}{lcc}
\hline ID & Hand squeezing & Tennis playing \\
\hline C1 & & + \\
C2 & + & + \\
C3 & + & + \\
C4 & + & - \\
C5 & + & + \\
C6 & - & + \\
C7 & - & + \\
C8 & + & + \\
C9 & - & + \\
C10 & + & + \\
Total percent (95\% exact Cl) & $70 \%(34.8-93.3)$ & $90 \%(55.5-99.7)$
\end{tabular}

$\mathrm{Cl}$, confidence interval; fMRI, functional magnetic resonance imaging.

TABLE 3 | Patient fMRI responses to hand squeeze and tennis motor imagery.

\begin{tabular}{|c|c|c|c|}
\hline ID & $\begin{array}{l}\text { LoC at } \\
\text { fMRI }\end{array}$ & Hand squeezing & Tennis playing \\
\hline $\mathrm{P} 1^{\mathrm{a}}$ & Coma & - & - \\
\hline$P 2^{a}$ & MCS+ & + & - \\
\hline$P 3^{a}$ & UWS & + & - \\
\hline$P 4^{a}$ & MCS- & - & + \\
\hline$P 5^{a}$ & MCS+ & + & - \\
\hline P6 & MCS+ & - & - \\
\hline P7 & UWS & - & + \\
\hline P8 & UWS & - & - \\
\hline P9 & MCS- & - & - \\
\hline P10 & UWS & - & - \\
\hline $\begin{array}{l}\text { Total percent }(95 \% \\
\text { exact Cl) }\end{array}$ & & $30 \%(6.7-65.3)$ & $20 \%(2.5-55.6)$ \\
\hline Sensitivity (95\% exact Cl) & & $66.7 \%(12.5-98.2)$ & $0 \%(0-70.8)$ \\
\hline Specificity (95\% exact Cl) & & $85.7 \%(42.1-99.6)$ & $71.4 \%(29.0-96.3)$ \\
\hline Accuracy & & $80 \%$ & $50 \%$ \\
\hline
\end{tabular}

Sensitivity, specificity, and accuracy of motor imagery paradigm detection of command following in patients who demonstrated behavioral evidence of command following.

fMRl, functional magnetic resonance imaging; LoC, level of consciousness; MCS-, minimally conscious state without language; MCS+, minimally conscious state with language; UWS, unresponsive wakefulness syndrome; Cl, confidence interval.

aThe patient was receiving sedatives at the time of data acquisition (see Table S3 in Supplementary Material for details). Image acquisition parameters for P10 differed from those of the other subjects (see Materials and Methods for details).

\section{Tennis Playing}

Nine of the 10 healthy subjects demonstrated command following via the tennis playing paradigm [90\% (55.5-99.7)]. Two of the 10 patients demonstrated command following on the tennis playing paradigm [20\% (2.5-55.6)]. Of the three patients with behavioral evidence of command following on bedside examination, none demonstrated command following on the tennis playing imagery paradigm $(\mathrm{TP}=0 / 3, \mathrm{FN}=3 / 3)$. Of the seven patients without behavioral evidence of command following, two demonstrated command following on the tennis playing motor imagery para$\operatorname{digm}(\mathrm{TN}=5 / 7, \mathrm{FP}=2 / 7)$. The sensitivity and specificity for the tennis playing paradigm for detecting behavioral evidence of command following were $0 \%(0-70.8)$ and $71.4 \%$ (29.0-96.3), respectively, and ACC $=50 \%$.

The agreement between presence or absence of command following via hand squeezing and tennis playing imagery was $60 \%$ in healthy subjects and $50 \%$ in patients. Data on the percentage of voxels activated for each subject in each paradigm are presented in Tables S2 and S4 in Supplementary Material for healthy subjects and patients, respectively. For both the hand squeeze and tennis imagery paradigms, at least one healthy subject demonstrated $0 \%$ activated voxels. Therefore, all patients with $>0 \%$ activated voxels in each paradigm met the predetermined criteria for having a positive response to the fMRI tasks (i.e., the ROI had $>0 \%$ activated voxels and the percentage of activated voxels in the ROI exceeded the 2.5th percentile of the normal range in healthy subjects).

\section{Effect of Sedation on Behavioral Diagnosis and fMRI Responses}

Administration of sedation $(n=5)$ was not associated with LoC or fMRI responses at the time of fMRI (Fisher's Exact Test, $\mathrm{df}=1$; $P=0.17-0.99$ for all analyses). The types and doses of sedative, anxiolytic, and analgesic medications administered at the time of fMRI are reported in Table S3 in Supplementary Material.

\section{DISCUSSION}

Precise assessment of LoC in patients with severe TBI is critical for guiding clinical management, providing accurate prognoses, and 
gaining access to rehabilitative services. Assessment of command following is a central component of the bedside examination and diagnostic impression. However, behavioral bedside assessment is susceptible to patient-, environment-, and examiner-related biases that contribute to the high misdiagnosis rate in this patient population. Multiple fMRI paradigms that probe covert consciousness have been investigated as potential objective markers of command following, but few studies have compared the utility of these paradigms concurrently in the same patient sample (31). Head-to-head comparison of fMRI command following tasks is needed to guide paradigm selection in future studies and eventually clinical practice. In addition, defining intrasubject variability would provide an objective basis for the cautious interpretation of absent fMRI responses to motor imagery paradigms. In this study, we assessed the sensitivity, specificity, and ACC of hand squeezing and tennis playing motor imagery paradigms in a sample of patients with severe TBI whose behavioral diagnosis at the time of the fMRI was coma, UWS, or MCS.

Although the tennis paradigm had higher ACC for detecting covert command following in healthy subjects, the hand squeezing paradigm accurately identified two of three patients who demonstrated behavioral evidence of command following (MCS+) as well as one patient (UWS) who did not. Conversely, responses to the tennis paradigm were absent in all three patients who demonstrated behavioral evidence of command following and present in two (MCS-, UWS) who did not. The sensitivity and sensitivity, specificity, and ACC of these findings suggest that the hand squeezing paradigm is a better classifier of command following in patients who are known to follow commands at the bedside than is the tennis playing paradigm.

One objective of developing fMRI paradigms for detection of conscious awareness in DoC is the prospect of identifying patients who retain the cognitive capacity for command following but do not demonstrate it at the bedside due to confounding factors such as impartments in speech or motor function. This cohort, described as having $\operatorname{CMD}(24,26)$, is at risk for early withdrawal of life-sustaining therapies and denial of access to rehabilitative care. Our tennis playing paradigm identified two such patients, neither of whom showed fMRI responses to the hand squeezing paradigm, while the hand squeezing paradigm identified one such patient. For the purpose of this study, which focused on identifying the fMRI paradigm that best detects command following and therefore tried to maximize the TPR, these patients were included in the "false positive" group when calculating specificity and ACC. However, rather than the fMRI findings being "false positives," it is possible that these three patients retained the cognitive capacity for command following but only demonstrated it on one of the two fMRI paradigms and not on bedside evaluation. Thus, the rate of detecting CMD was higher for the tennis playing paradigm than hand squeezing, even though the hand squeezing paradigm identified patients with behavioral evidence of command following with a higher ACC than did tennis. Future studies should address this apparent discrepancy between overall ACC and CMD detection rate by increasing the sample size, increasing the number of experimental runs, and interleaving the presentation of the fMRI paradigms. When possible, administering more than one type of command following paradigm should be considered to maximize the probability of detecting conscious awareness.

The tennis playing paradigm performed better than the hand squeezing task in the healthy control group while the opposite was true for patients. Comparison of fMRI activation profiles between healthy subjects and brain-injured patients must be performed with caution, given the multitude of factors that may influence an individual's fMRI response. For example, in healthy subjects, playing tennis, whether on the court or in one's imagination, may be a more cognitively challenging and salient task compared to a mundane hand-squeezing task. Conversely, hand squeezing may become more salient in a brain-injured patient who is asked to perform this task frequently during neurological examinations in the ICU. Furthermore, it is likely that imagining playing tennis requires multimodal processing and therefore would be expected to evoke a more distributed network than the unimodal task of imagining squeezing one's hand. If so, patients, as compared to healthy subjects, may have access to less of the distributed network required to mediate tennis imagery due to focal lesions and loss of connectivity. For those patients who are able to cognitively perform the task, the frequent repetition of the command in the clinical environment may be associated with a mental training effect and/or an increased effort applied to the task and hence a more robust fMRI response (45). It is also possible that the patients who followed commands at the bedside were actually squeezing their hand in the scanner, rather than imagining the movement, leading to more robust SMA/PMC activation. In this study, we did not systematically record hand movements during the scanning session. Future studies that compare fMRI paradigms in this patient population should consider incorporating visual or electromyographic monitoring into their paradigm.

Several limitations should be considered when interpreting the results of this study. First, although this is one of the only studies that compares the ACC of fMRI motor imagery paradigms in a patient cohort, the sample size is small and includes patients in both acute and chronic phases of recovery. Consequently, generalizing our findings to other patient groups should be done with caution and future investigations should consider larger sample sizes of more homogenous patient groups enrolled across multiple sites. Second, the CIs around the fMRI command following proportions are very wide, suggesting that our sample has high variability and that the findings may lack precision when compared to the overall population of patients with traumatic DoC. Third, at the time of the fMRI scan, several subjects were receiving pharmacological sedation. Sedatives were administered at the discretion of the treating clinicians for patient safety or comfort and therefore could not be lifted for this study. Indeed, even patients who are comatose may require sedation to treat bronchospasm caused by an endotracheal tube. Sedation is therefore a medical necessity for some critically ill patients, and it is therefore unlikely that any fMRI study of critically ill patients that aims to be generalizable will be able to exclude patients receiving sedation.

Although the effects of sedating agents on cortical responses (46) and connectivity (47) are beginning to be elucidated, the impact of these pharmacological interventions may vary with multiple patient-specific factors, including tolerance, body mass, 
and metabolism. Furthermore, standardized sedation rating scales that have been validated in non-brain injured critically ill patients (e.g., the Richmond Agitation Sedation Scale) (48) are not applicable in severely brain-injured patients, for whom the behavioral effects of sedation cannot be quantitatively distinguished from the behavioral effects of the brain injury itself. As a result, we could not quantitatively measure the effect of sedation for each patient prior to scanning. Therefore, despite the lack of a statistical association between sedation and fMRI responsiveness, it is still possible that sedation affected the fMRI responses, though the results of the hand and tennis motor imagery paradigms would have been affected equally as all data were collected during the same scanning session.

Finally, we could not avoid some methodological challenges such as the hand squeezing motor imagery task always preceding the tennis playing motor imagery task. Though our findings do not suggest that this fixed order contributed to systematically poor arousal and therefore decreased fMRI responses to the tennis playing paradigm, future studies should consider randomizing the presentation of the tasks. In addition, imaging parameters for one patient, who did not show responses to either paradigm, did not conform to those of the other subjects, potentially adding variability to the data. The examiner completing the behavioral assessments for this study was not blinded to the previously determined clinical diagnosis of the patient (i.e., the diagnosis of LoC rendered by the treating physicians and nurses) and the investigator conducting the imaging analysis was not blinded to the behavioral assessment. Therefore, it is possible that the clinical diagnosis biased the behavioral assessment reported here and that, despite the automated nature of the imaging analysis, the behavioral assessment influenced the fMRI results. To avoid this potential limitation in the future, the behavioral assessment completed for study purposes should be conducted by an examiner who is not involved in the clinical care of the patient or in screening patients for inclusion in the study and the investigator conducting the imaging analysis should be blinded to the behavioral diagnosis. Finally, despite recent evidence supporting the need for serial behavioral assessment of LoC to improve diagnostic accuracy (16), we were only able to administer one CRS-R prior to the fMRI study due to the medical instability of the acutely ill patients and the limited time available to examine chronic patients returning for clinical follow-up. Future studies should aim to administer multiple behavioral assessments to establish the diagnostic baseline.

It is important to note that there was poor agreement between fMRI responses occurring during the hand squeezing and tennis playing fMRI squeezing tasks in both healthy subjects and patients. Specifically, all the healthy subjects who failed to demonstrate an fMRI response to hand squeezing or tennis imagery did show an fMRI response to the other paradigm. All of the patients who demonstrated an fMRI response to hand squeezing or tennis playing failed to show an fMRI response to the other paradigm. Fluctuation in arousal, task characteristics, prior exposure to the sport, and intersubject variability may have contributed to the inconsistencies in these findings. Future studies could consider individualizing the task such that it matches each participant's experiences or interviewing participants and surrogates to ascertain prior exposure.

In choosing the appropriate task for detecting consciousness in patients diagnosed with DoC, investigators should carefully consider the research aims because a series of decisions (e.g., paradigm, analytic pipeline, and interpretation algorithm) related to the specific question of interest may alter the study design and findings. Furthermore, factors unrelated to cognitive ability, such as subject familiarity with the imagined task (e.g., a patient who has played tennis may be more responsive than a patient who has only watched the game on television) and analytic strategies (e.g., objective application of a priori ROIs versus subjective reading of fMRI activation maps) may introduce uncertainty into the data. Thus, different fMRI paradigms may lead to variable results in the same patient. We found that in patients with severe TBI diagnosed with DoC, the hand squeezing motor imagery paradigm detected covert command following with greater ACC than the tennis playing paradigm. However, the tennis playing paradigm was more sensitive in healthy subjects and identified more patients with CMD. These findings should be considered hypothesis-generating and will require replication in a larger sample of patients across multiple clinical and research sites. Clinical implementation of fMRI motor imagery paradigms for detection of consciousness will require further development, validation, and optimization of standardized approaches to fMRI data acquisition, analysis and interpretation.

\section{ETHICS STATEMENT}

This study was carried out in accordance with a protocol approved by the Partners Institutional Review Board. Patient surrogate decision-makers gave written informed consent in accordance with the Declaration of Helsinki.

\section{AUTHOR CONTRIBUTIONS}

The authors contributed to the following aspects of the study: YB, JG, and BE: substantial contributions to the conception or design of the work; or the acquisition, analysis, or interpretation of data for the work; drafting the work or revising it critically for important intellectual content; final approval of the version to be published; and agreement to be accountable for all aspects of the work in ensuring that questions related to the accuracy or integrity of any part of the work are appropriately investigated and resolved.

\section{ACKNOWLEDGMENTS}

The authors thank the nursing staffs of the Massachusetts General Hospital Neurosciences ICU, Multidisciplinary ICU, and the Surgical ICU. We also thank Kellie Cahill and the MRI technologists for their assistance with data acquisition. We would like to acknowledge Dylan Tisdall and Andre van der Kouwe (Athinoula A Martinos Center for Biomedical Imaging) and Himanshu Bhat (Siemens Medical Center) for the provision of WIP711D (vNav Motion-Corrected Multiecho MPRAGE) used to acquire MEMPRAGE data. We are grateful to the patients and 
families in this study for their participation and support. This work was supported by grants from the National Institutes of Health (K23NS094538), the Center for Integration of Medicine \& Innovative Technology (Boston, MA, USA), the American Academy of Neurology/American Brain Foundation, the James S. McDonnell Foundation, the Massachusetts General Hospital Department of Neurology and Division of Neurocritical Care and Emergency Neurology, and the National Institute on

\section{REFERENCES}

1. Jennett B, Plum F. Persistent vegetative state after brain damage. A syndrome in search of a name. Lancet (1972) 1(7753):734-7. doi:10.1016/ S0140-6736(72)90242-5

2. Giacino JT, Kalmar K. Diagnostic and prognostic guidelines for the vegetative and minimally conscious states. Neuropsychol Rehabil (2005) 15(3-4):166-74. doi:10.1080/09602010443000498

3. Laureys S, Celesia GG, Cohadon F, Lavrijsen J, Leon-Carrrion J, Sannita WG, et al. Unresponsive wakefulness syndrome: a new name for the vegetative state or apallic syndrome. BMC Med (2010) 8(1):68. doi:10.1186/17417015-8-68

4. Giacino JT, Ashwal S, Childs N, Cranford R, Jennett B, Katz DI, et al. The minimally conscious state: definition and diagnostic criteria. Neurology (2002) 58(3):349-53. doi:10.1212/WNL.58.3.349

5. Nakase-Thompson R, Sherer M, Yablon SA, Nick TG, Trzepacz PT. Acute confusion following traumatic brain injury. Brain Inj (2004) 18(2):131-42. doi:10.1080/0269905031000149542

6. Sherer M, Nakase-Thompson R, Yablon SA, Gontkovsky ST. Multidimensional assessment of acute confusion after traumatic brain injury. Arch Phys Med Rehabil (2005) 86(5):896-904. doi:10.1016/j.apmr.2004.09.029

7. Hirschberg R, Giacino JT. The vegetative and minimally conscious states: diagnosis, prognosis and treatment. Neurol Clin (2011) 29(4):773-86. doi:10.1016/j.ncl.2011.07.009

8. Nakase-Richardson R, Whyte J, Giacino JT, Pavawalla S, Barnett SD, Yablon SA, et al. Longitudinal outcome of patients with disordered consciousness in the NIDRR TBI model systems programs. J Neurotrauma (2012) 29(1):59-65. doi:10.1089/neu.2011.1829

9. Whyte J, Nakase-Richardson R, Hammond FM, McNamee S, Giacino JT, Kalmar K, et al. Functional outcomes in traumatic disorders of consciousness: 5 -Year outcomes from the national institute on disability and rehabilitation research traumatic brain injury model systems. Arch Phys Med Rehabil (2013) 94(10):1855-60. doi:10.1016/j.apmr.2012.10.041

10. Giacino JT, Kalmar K. The vegetative and minimally conscious states: a comparison of clinical features and functional outcome. J Head Trauma Rehabil (1997) 12(4):36-51. doi:10.1097/00001199-199708000-00005

11. Wannez S, Hoyoux $\mathrm{T}$, Langohr $\mathrm{T}$, Bodart $\mathrm{O}$, Martial $\mathrm{C}$, Wertz J, et al. Objective assessment of visual pursuit in patients with disorders of consciousness: an exploratory study. J Neurol (2017) 264(5):928-37. doi:10.1007/ s00415-017-8469-0

12. Schnakers C, Vanhaudenhuyse A, Giacino J, Ventura M, Boly M, Majerus S, et al. Diagnostic accuracy of the vegetative and minimally conscious state: clinical consensus versus standardized neurobehavioral assessment. BMC Neurol (2009) 9:35. doi:10.1186/1471-2377-9-35

13. Andrews K, Murphy L, Munday R, Littlewood C. Misdiagnosis of the vegetative state: retrospective study in a rehabilitation unit. BMJ (1996) 313(7048):13-6. doi:10.1136/bmj.313.7048.13

14. Childs NL, Mercer WN, Childs HW. Accuracy of diagnosis of persistent vegetative state. Neurology (1993) 43(8):1465-7. doi:10.1212/WNL.43.8.1465

15. Kalmar K, Giacino JT. The JFK coma recovery scale - revised. Neuropsychol Rehabil (2005) 15(3-4):454-60. doi:10.1080/09602010443000425

16. Wannez S, Heine L, Thonnard M, Gosseries O, Laureys S; Coma Science Group Collaborators. The repetition of behavioral assessments in diagnosis of disorders of consciousness: repeated CRS-R assessments for diagnosis in DOC. Ann Neurol (2017) 81(6):883-9. doi:10.1002/ana.24962
Disability, Independent Living, and Rehabilitation Research (NIDILRR).

\section{SUPPLEMENTARY MATERIAL}

The Supplementary Material for this article can be found online at http://www.frontiersin.org/articles/10.3389/fneur.2017.00688/ full\#supplementary-material.

17. Monti MM, Vanhaudenhuyse A, Coleman MR, Boly M, Pickard JD, Tshibanda L, et al. Willful modulation of brain activity in disorders of consciousness. N Engl J Med (2010) 362(7):579-89. doi:10.1056/NEJMoa0905370

18. Coleman MR, Bekinschtein T, Monti MM, Owen AM, Pickard JD. A multimodal approach to the assessment of patients with disorders of consciousness. Prog Brain Res (2009) 177:231-48. doi:10.1016/S0079-6123(09)17716-6

19. Owen AM, Coleman MR, Boly M, Davis MH, Laureys S, Pickard JD. Detecting awareness in the vegetative state. Science (2006) 313(5792):1402. doi:10.1126/ science.1130197

20. Coleman MR, Davis MH, Rodd JM, Robson T, Ali A, Owen AM, et al. Towards the routine use of brain imaging to aid the clinical diagnosis of disorders of consciousness. Brain (2009) 132(9):2541-52. doi:10.1093/brain/awp183

21. Rodriguez Moreno D, Schiff ND, Giacino J, Kalmar K, Hirsch J. A network approach to assessing cognition in disorders of consciousness. Neurology (2010) 75(21):1871-8. doi:10.1212/WNL.0b013e3181feb259

22. Di H, Boly M, Weng X, Ledoux D, Laureys S. Neuroimaging activation studies in the vegetative state: predictors of recovery? Clin Med (Lond) (2008) 8(5):502-7. doi:10.7861/clinmedicine.8-5-502

23. Giacino JT, Hirsch J, Schiff N, Laureys S. Functional neuroimaging applications for assessment and rehabilitation planning in patients with disorders of consciousness. Arch Phys Med Rehabil (2006) 87(12 Suppl 2):S67-76. doi:10.1016/j.apmr.2006.07.272

24. Edlow BL, Chatelle C, Spencer CA, Chu CJ, Bodien YG, O'Connor KL, et al. Early detection of consciousness in patients with acute severe traumatic brain injury. Brain (2017) 140(9):2399-414. doi:10.1093/brain/awx176

25. Schiff ND, Rodriguez-Moreno D, Kamal A, Kim KHS, Giacino JT, Plum F, et al. fMRI reveals large-scale network activation in minimally conscious patients. Neurology (2005) 64(3):514-23. doi:10.1212/01.WNL.0000150883.10285.44

26. Schiff ND. Cognitive motor dissociation following severe brain injuries. JAMA Neurol (2015) 72(12):1413. doi:10.1001/jamaneurol.2015.2899

27. Naci L, Owen AM. Making every word count for nonresponsive patients. JAMA Neurol (2013) 70(10):1235-41. doi:10.1001/jamaneurol.2013.3686

28. Rossetti AO, Laureys S. Clinical Neurophysiology in Disorders of Consciousness Brain Function Monitoring in the ICU and Beyond [Internet]. Vienna: Springer (2015). Available from: http://ezproxy.unav.es:2048/login?url=http://link. springer.com/book/

29. Laureys S, Schiff ND. Coma and consciousness: paradigms (re)framed by neuroimaging. Neuroimage (2012) 61(2):478-91. doi:10.1016/j.neuroimage. 2011.12.041

30. Boly M, Coleman MR, Davis MH, Hampshire A, Bor D, Moonen G, et al. When thoughts become action: an fMRI paradigm to study volitional brain activity in non-communicative brain injured patients. Neuroimage (2007) 36(3):979-92. doi:10.1016/j.neuroimage.2007.02.047

31. Gibson RM, Fernndez-Espejo D, Gonzalez-Lara LE, Kwan BY, Lee DH, Owen AM, et al. Multiple tasks and neuroimaging modalities increase the likelihood of detecting covert awareness in patients with disorders of consciousness. Front Hum Neurosci (2014) 8:950. doi:10.3389/fnhum. 2014.00950

32. Cruse D, Chennu S, Chatelle C, Bekinschtein TA, Fernández-Espejo D, PickardJD, et al. Bedside detection of awareness in the vegetative state: a cohort study. Lancet (2011) 378(9809):2088-94. doi:10.1016/S0140-6736(11)61224-5

33. Cruse D, Chennu S, Chatelle C, Fernandez-Espejo D, Bekinschtein TA, Pickard JD, et al. Relationship between etiology and covert cognition in the minimally conscious state. Neurology (2012) 78(11):816-22. doi:10.1212/ WNL.0b013e318249f764 
34. Giacino JT, Kalmar K, Whyte J. The JFK coma recovery scale-revised: measurement characteristics and diagnostic utility. Arch Phys Med Rehabil (2004) 85(12):2020-9. doi:10.1016/j.apmr.2004.02.033

35. Bruno M-A, Majerus S, Boly M, Vanhaudenhuyse A, Schnakers C, Gosseries O, et al. Functional neuroanatomy underlying the clinical subcategorization of minimally conscious state patients. JNeurol (2012) 259(6):1087-98. doi:10.1007/s00415-011-6303-7

36. Bruno M-A, Vanhaudenhuyse A, Thibaut A, Moonen G, Laureys S. From unresponsive wakefulness to minimally conscious PLUS and functional lockedin syndromes: recent advances in our understanding of disorders of consciousness. J Neurol (2011) 258(7):1373-84. doi:10.1007/s00415-011-6114-x

37. van der Kouwe AJW, Benner T, Salat DH, Fischl B. Brain morphometry with multiecho MPRAGE. Neuroimage (2008) 40(2):559-69. doi:10.1016/j. neuroimage.2007.12.025

38. Beckmann CF, Jenkinson M, Smith SM. General multilevel linear modeling for group analysis in FMRI. Neuroimage (2003) 20(2):1052-63. doi:10.1016/ S1053-8119(03)00435-X

39. Woolrich MW, Behrens TEJ, Beckmann CF, Jenkinson M, Smith SM. Multilevel linear modelling for FMRI group analysis using Bayesian inference. Neuroimage (2004) 21(4):1732-47. doi:10.1016/j.neuroimage. 2003.12.023

40. Eklund A, Nichols TE, Knutsson H. Cluster failure: why fMRI inferences for spatial extent have inflated false-positive rates. Proc Natl Acad Sci U S A (2016) 113(28):7900-5. doi:10.1073/pnas.1602413113

41. Eickhoff SB, Stephan KE, Mohlberg H, Grefkes C, Fink GR, Amunts K, et al. A new SPM toolbox for combining probabilistic cytoarchitectonic maps and functional imaging data. Neuroimage (2005) 25(4):1325-35. doi:10.1016/j. neuroimage.2004.12.034

42. Fernández-Espejo D, Junqué C, Vendrell P, Bernabeu M, Roig T, Bargalló N, et al. Cerebral response to speech in vegetative and minimally conscious states after traumatic brain injury. Brain Inj (2008) 22(11):882-90. doi:10.1080/02699050802403573
43. Bardin JC, Fins JJ, Katz DI, Hersh J, Heier LA, Tabelow K, et al. Dissociations between behavioural and functional magnetic resonance imaging-based evaluations of cognitive function after brain injury. Brain (2011) 134(3):769-82. doi:10.1093/brain/awr005

44. StataCorp. Stata Statistical Software: Release. College Station, TX: StatCorp LP (2015).

45. Kami A, Meyer G, Jezzard P, Adams MM, Turner R, Ungerleider LG. Functional MRI evidence for adult motor cortex plasticity during motor skill learning. Nature (1995) 377(6545):155-8. doi:10.1038/377155a0

46. Davis MH, Coleman MR, Absalom AR, Rodd JM, Johnsrude IS, Matta BF, et al. Dissociating speech perception and comprehension at reduced levels of awareness. Proc Natl Acad Sci U S A (2007) 104(41):16032-7. doi:10.1073/ pnas.0701309104

47. Kirsch M, Guldenmund P, Ali Bahri M, Demertzi A, Baquero K, Heine L, et al. Sedation of patients with disorders of consciousness during neuroimaging: effects on resting state functional brain connectivity. Anesth Analg (2017) 124(2):588-98. doi:10.1213/ANE.0000000000001721

48. Sessler CN, Gosnell MS, Grap MJ, Brophy GM, O’Neal PV, Keane KA, et al. The Richmond Agitation-Sedation Scale: validity and reliability in adult intensive care unit patients. Am J Respir Crit Care Med (2002) 166(10):1338-44. doi: $10.1164 / \mathrm{rccm} .2107138$

Conflict of Interest Statement: The authors declare that the research was conducted in the absence of any commercial or financial relationships that could be construed as a potential conflict of interest.

Copyright (c) 2017 Bodien, Giacino and Edlow. This is an open-access article distributed under the terms of the Creative Commons Attribution License (CC BY). The use, distribution or reproduction in other forums is permitted, provided the original author(s) or licensor are credited and that the original publication in this journal is cited, in accordance with accepted academic practice. No use, distribution or reproduction is permitted which does not comply with these terms. 